chercher : repérer : avancer

Cet article est disponible en ligne à l'adresse :

http://www.cairn.info/article.php?ID_REVUE=PARL\&ID_NUMPUBLIE=PARL_008\&ID_ARTICLE=PARL $008 \quad 0085$

Étudiants et politique en Italie (1945-1968)

par Giovanni ORSINA et Frédéric ATTAL

| L'Harmattan | Parlement[s]

$2007 / 2-N^{\circ} 8$

ISSN 1768-6520 | pages 85 à 102

Pour citer cet article :

-Orsina G. et Attal F., Étudiants et politique en Italie (1945-1968), Parlement[s] 2007/2, N 8, p. 85-102.

Distribution électronique Cairn pour L'Harmattan.

(C) L'Harmattan. Tous droits réservés pour tous pays.

La reproduction ou représentation de cet article, notamment par photocopie, n'est autorisée que dans les limites des conditions générales d'utilisation du site ou, le cas échéant, des conditions générales de la licence souscrite par votre établissement. Toute autre reproduction ou représentation, en tout ou partie, sous quelque forme et de quelque manière que ce soit, est interdite sauf accord préalable et écrit de l'éditeur, en dehors des cas prévus par la législation en vigueur en France. Il est précisé que son stockage dans une base de données est également interdit. 


\section{Étudiants et politique en Italie (1945-1968)}

\section{Giovanni Orsina}

Professeur associé à l'université Luiss-Guido-Carli de Rome

Traduit de l’italien par Frédéric Attal

Le présent essai entend proposer quelques hypothèses d'interprétation historiographique des relations qu'entretiennent les étudiants des universités italiennes avec la politique, le système politique et les partis « adultes » durant les vingt années qui séparent l'après-guerre de 1968. Les associations et instances représentatives étudiantes furent durant ces années une composante essentielle de l'espace public italien. D'abord, parce qu'elles ont, dans de nombreux cas, joué le rôle d'avantgarde idéologique et de champ d'expérimentation politique; puis, parce qu'elles ont représenté un important creuset de sélection et de formation 
de la classe dirigeante ${ }^{1}$; ensuite, parce que la vie publique universitaire fut l'un des lieux, et non des moindres, où, durant les vingt premières années de la République, les partis allèrent à la rencontre de la société politique et de la société civile, - l'étudier signifie alors observer, d'un point de vue partiel mais original, l'évolution du système de parti, en analysant comment celui-ci a réussi à contrôler des processus qui lui étaient étrangers ; enfin, parce que réfléchir sur la crise mortelle des associations et des instances représentatives étudiantes universitaires peut nous aider à comprendre la nature particulière du 1968 italien.

Je résumerai en ces termes l'ensemble de ma démonstration. Dans la première moitié des années 1950, la vie publique des étudiants a trouvé un mode de fonctionnement très proche du système politique parlementaire, en ce sens que des associations idéologiquement marquées et se faisant concurrence coexistaient à l'intérieur d'organismes représentatifs élus par les étudiants. Durant la période qui les a amenés à s'organiser ainsi, les étudiants - sur l'impulsion surtout des associations 《goliardiques » ${ }^{2}$ - ont élaboré un patrimoine idéologique qui exaltait surtout l'indépendance d'esprit, la capacité d'innovation et la créativité de la jeunesse. En bref, leur politique, selon ce schéma, ne devrait pas être pour les étudiants l'émanation des "adultes», mais une construction autonome, adaptée à leurs exigences spécifiques.

À partir de la moitié des années 1950 cependant, en raison notamment de l'évolution globale du système italien des partis, les logiques et les priorités de la vie publique universitaire se sont de plus en plus rapprochées des logiques et des priorités de la politique nationale, jusqu'à coïncider grosso modo avec celles-ci, et les rapports entre les partis et les associations étudiantes sont devenus de plus en plus étroits. Mais cette transformation ne s'est pas accompagnée d'une révision parallèle des bases idéologiques fondamentales de l'associationnisme étudiant, qui a continué à se réclamer des principes autonomistes de ses débuts. Entre le discours rhétorique de la politique étudiante et sa réalité s'ouvrit ainsi brusquement un hiatus croissant, correspondant sous de nombreux aspects à celui qui, au début des années 1960, était en train de se créer au niveau national, entre l'affirmation d'une culture antifasciste et

\footnotetext{
${ }^{1}$ Ont participé à la vie publique universitaire, à titre d'exemples : l'actuel Président de la République Giorgio Napolitano, l'actuel vice-coordonnateur de Forza Italia Fabrizio Cicchito, le leader radical Marco Pannella, l'ancien leader socialiste et président du Conseil Bettino Craxi, ou encore des dirigeants d'entreprises publiques ou de la télévision.

2 Traduction littérale de l'italien « goliardico » qui désigne des associations estudiantines à but corporatiste attachées à maintenir la tradition étudiante.
} 
progressiste encline à relancer les promesses d'une palingénésie de la Résistance, et les équilibres de pouvoir, plutôt modérés, en voie de consolidation. C'est dans ce hiatus que s'enracinèrent les courants étudiants activistes radicaux, enclins à critiquer les associations et les organismes représentatifs traditionnels, précisément au nom de l'idéologie autonomiste, depuis toujours à la base de leur légitimation.

Ces courants constitueraient enfin une grande partie de la contestation de 68 qui, pour ces raisons, n'a pas seulement été une rébellion contre le système politique «adulte» et les institutions étudiantes, mais fut aussi, idéologiquement, sous bien des aspects, l'enfant de ce système et de ces institutions.

\section{Les années 1950 : le temps de la singularité}

Alors que l'Italie retrouvait la démocratie, le monde étudiant était traversé par deux tensions contradictoires : d'un côté, il voulait participer à la reconstruction de la vie publique nationale, en s'engageant dans la politique et en acceptant alors que se reflètent en son sein les divisions partisanes présentes chez les «adultes »; de l'autre, il désirait au contraire préserver une ancienne tradition d'autonomie, d'unité et de solidarité. La politisation et la fragmentation idéologique conséquente de la communauté universitaire se présentaient depuis l'immédiat après-guerre comme un fait qu'il était difficile d'ignorer: socialistes et communistes, catholiques, corporatistes étudiants (goliardi), néofascistes entrainaient les étudiants vers des rivages différents et strictement incompatibles. Dans le même temps, chacun de ces groupes pensait qu'il était indispensable de recomposer la communauté étudiante dans un cadre unitaire: mais comment cette unité devait-elle être conçue ? C'est une question qui trouvait autant de réponses qu'il y avait de positions idéologiques. Pour les socialistes et communistes du Front de la jeunesse, la cohésion étudiante représentait à peu près la transposition dans le champ universitaire de l'espoir qu'ils nourrissaient d'un ample rassemblement démocratique, et qu'ils tentaient de réaliser aussi dans l'arène publique nationale. Dans un cadre "professionnellement» circonscrit comme celui des campus, la solution frontiste prenait une coloration syndicale absente au niveau des grandes instances politiques; il s'agissait toutefois d'un syndicalisme instrumental et subordonné à l'utilisation politique et propagandiste que les socialistes et les communistes pouvaient en faire. La Fédération universitaire catholique italienne (FUCI) regardait avec méfiance le modèle de vie publique universitaire proposé et soutenu par la gauche marxiste, en en craignant surtout les implications politiques. La 
forme d'unité estudiantine que les catholiques acceptèrent finalement fut alors subordonnée à la condition que seuls pouvaient s'y agréger les « étudiants en tant que tels », c'est-à-dire porteurs d'intérêts catégoriels spécifiques, d'ordre technique et corporatiste, et non d'objectifs et de valeurs aux implications politiques plus vastes et générales. Les goliardi enfin revendiquaient l'idée d'unité de leurs condisciples, selon les anciennes traditions des campus italiens dont ils affirmaient vouloir s'inspirer jusque pour leur nom. Pour certains d'entre eux, cette unité venait nécessairement du fait que devait prévaloir la qualité essentielle d'étudiant sur toute forme de division idéologique, et impliquait ainsi le refus radical de la politique au nom du droit des jeunes au désengagement. D'autres pensaient au contraire que les étudiants devaient participer unis à la transformation de l'université en une communauté démocratique qui, partant de sa propre identité, appuie et soutienne le processus plus ambitieux de construction de la démocratie républicaine alors en marche.

Cette situation rendait impossible la reconstruction du corps étudiant dans une forme unitaire, sinon en en reconnaissant les profondes fractures internes, ou bien en bâtissant des institutions aptes à consentir la pleine expression du pluralisme idéologique. La solution qui fut finalement trouvée admettait un double niveau d'organisation: d'un côté, les associations «fermées », plurielles, partisanes et idéologiquement orientées; de l'autre, des organes représentatifs unitaires et "universels», élus au niveau de chaque campus (Organismes représentatifs) comme au niveau national (Union nationale universitaire représentative italienne, UNURI), entretenant avec les associations fermées le type de relations que les partis politiques entretenaient avec le Parlement. Il était parfaitement évident que les organismes représentatifs avaient aussi pour tâche d'exprimer les revendications corporatives des étudiants. Cette dimension syndicale resta toutefois dans une large mesure subordonnée à la sphère politique, souvent même instrumentalisée par celle-ci. En bref, l'organisation "parlementaire » et «partisane » que les étudiants se donnèrent entre la fin des années 1940 et le début des années 1950, bien que destinée à être revue et rediscutée par toutes les générations qui se succédèrent ensuite à la tête des institutions universitaires, donnerait malgré tout à l'histoire de l'UNURI et des associations une empreinte essentiellement politique dont elle ne se débarrasserait jamais.

Les jeunes de l'Union goliardique italienne (UGI) jouèrent un rôle de premier plan, dans la première moitié des années 1950, pour définir les caractères que devait prendre la vie publique étudiante. Cette première 
génération de goliardisme "politique» proposa à la communauté universitaire une voie de développement originale qui devait lui permettre de participer de façon autonome, et en respectant sa nature spécifique, au processus de construction de la démocratie républicaine, et, en même temps, qui rendait possible les aspirations unitaires diffuses dans les campus et coexistant avec la profonde fragmentation idéologique. Les étudiants, selon les goliardi, devaient s'affirmer comme acteurs indépendants d'un projet politique, en veillant à ajuster leur projet aux exigences particulières de la réalité universitaire. De cette manière, ils pouvaient faire de la politique en évitant de s'enfermer dans le corporatisme et de rompre le lien avec la vie publique italienne, tout en restant fidèles à leur vocation propre et à la spécificité de leur milieu. Ils apprenaient soit à affronter et à résoudre les problèmes concrets de leur condition d'étudiants, soit à coordonner des activités communes en suivant des procédures respectueuses du pluralisme et en acceptant les diversités idéologiques. À l'intérieur de ce cadre général, les goliardi devaient ensuite assumer la fonction spécifique d'éducateurs : ils devaient convaincre, exhorter, forcer si nécessaire les étudiants communistes, fascistes, catholiques, ou simplement apolitiques, à accepter la démocratie universitaire. Ils construisaient ainsi un espace publique jeune, non seulement autonome par rapport à celui des «adultes », mais encore meilleur, plus libre, créatif, dynamique, posant ainsi les bases pour « reconstruire l'unité de la nation à partir de l'unité d'une génération ».

En réfléchissant sur les caractères, les formes et les buts de la vie représentative universitaire, les jeunes de l'UGI ne pouvaient certes pas ne pas affronter la difficile question des rapports entre associations étudiantes et partis «adultes». La tradition goliardique de transgression, de non-engagement et de net refus de tout ce qui pouvait risquer de rompre l'unité existentielle et générationnelle des étudiants, poussait les goliardi à répéter le mot d'ordre : «les partis, hors des universités! ». Le désir de participer au processus de reconstruction de la démocratie italienne, et le choix conséquent d'organiser les étudiants suivant des logiques politiques, rendaient toutefois cette position particulièrement difficile à tenir. Ce d'autant plus que la présence des partis dans les campus était, après-guerre, une réalité dont il fallait nécessairement tenir compte. Les goliardi trouvèrent à ce problème une solution aussi brillante que difficile à mettre en pratique : en voulant contribuer à la construction de la démocratie républicaine, les étudiants devaient faire de la politique ; mais ils devaient aussi respecter la spécificité de la vie universitaire, et défendre de ce fait, contre toute ingérence extérieure, l'indépendance et l'originalité de leur projet. Les associations étudiantes devaient en somme 
devenir des sujets politiques autonomes, capables de traiter avec les partis «adultes » à égalité, voire capables souvent de leur imposer leur intransigeance démocratique, leur désir de rénovation nationale, le refus propre à la jeunesse de la lâcheté, des hypocrisies, des schémas tout faits.

La génération des goliardi qui, à la tête de l'UGI dans la première moitié des années 1950, avait élaboré cette conception politique mais non partisane de l'engagement étudiant, réussit aussi à la réaliser, et à influencer de ses propres choix les autres associations étudiantes et la vie des instances représentatives. Tandis que les Centres universitaires démocratiques (CUDI) étaient durant ces années l'émanation étudiante du Parti socialiste et surtout du Parti communiste, que le Front universitaire d'action nationale (FUAN) était l'avatar du néofasciste Mouvement social italien, et que l'Entente réunissait en son sein un monde catholique divers qui, sur le plan politique, se réclamait de la Démocratie chrétienne, l'Union goliardique avait sans conteste construit un rapport particulier avec les partis dits laïques - républicain, et surtout libéral et social-démocrate. Cela dit, jusqu'à la fin des années 1950, l'UGI réussit à garder son autonomie. Souvent même, la perspective finissait par être renversée, et les goliardi tentaient d'amener à l'intérieur des partis respectifs les ambitieux desseins de rénovation nationale qu'ils avaient élaborés et dont ils s'étaient imprégnés au sein de l'Union. Les appartenances et les convergences générationnelles prévalaient somme toute sur les appartenances et les divergences politiques. Et il était de l'intérêt de tous que l'Union fût un lieu de rencontre autonome et créatif entre des individus libres qui, par la suite, s'ils le désiraient, pouvaient également s'engager individuellement dans les partis.

\section{Les années 1960 : le temps des partis}

Préserver l'Union goliardique, espérer amener l'ensemble de l'organisation de la vie étudiante à s'engager résolument en politique et, dans le même temps, à garder une autonomie jalouse non seulement par rapport aux partis, mais encore à l'égard de toute la structure idéologique et constitutionnelle "adulte», devenaient cependant des missions presque impossibles durant cette période intermédiaire. Une fois le choix fait de mobiliser les étudiants sur le terrain politique, faire face au monde extérieur, hors des campus, devenait inévitable. Que la relation entre étudiants et partis se place sur un plan d'égalité et d'autonomie ou, qu'au contraire, la vie publique des étudiants doive en fin de compte se conformer aux logiques et aux priorités de la politique "adulte», cela relevait en premier lieu de la conscience des jeunes. Plus exactement, cela 
dépendait de ce qui allait prévaloir dans l'esprit de ces jeunes: la solidarité générationnelle ou celle du parti ? En second lieu, cela relevait avant tout du contexte historique. C'est parce que l'UGI avait représenté le principal centre de réflexion sur l'engagement politique des étudiants et sur leurs rapports avec les partis «adultes» que le processus de transformation qui la concerne entre la fin des années 1950 et le début des années 1960 peut être considéré comme caractéristique des mutations plus générales qui durant ces années ont amené l'espace public étudiant à coïncider dans une large mesure avec celui des « adultes».

Tout en étant assez jaloux de leur autonomie, les goliardi avaient créé dans la première moitié des années 1950 des rapports de proximité particulière avec les partis laïques. Or, dans une situation comme celle de la première législature républicaine (1948-1953), caractérisée par la nette hégémonie de la Démocratie chrétienne au Parlement et par un climat international de dur affrontement entre les blocs, il n'était pas politiquement impossible d'envisager une convergence entre les étudiants républicains, libéraux et sociaux-démocrates en une seule organisation qui se donnât comme buts élevés et ambitieux d'éduquer les jeunes générations aux valeurs constitutionnelles ou de participer à la sécularisation du catholicisme politique. En bref, il n'était pas impossible d'imaginer que l'UGI parvînt à rester « si socialiste qu'elle ne puisse être libérale, si libérale qu'elle ne puisse être socialiste », comme on le disait à l'époque. À partir de la seconde législature toutefois, quand échoue le mécanisme électoral majoritaire introduit pour les élections de 1953, le panorama de la vie publique italienne commença à changer en profondeur. Et avec les années, les effets de cette transformation se firent sentir également, et pesamment, dans les campus. Comme l'écrirait bien après l'un des protagonistes de la vie universitaire de ces années, Paolo Ungari, la « plus vaste crise des autonomies de la jeunesse » à la fin des années 1950 doit être reliée « au déclin de l'équilibre éthique et idéal propre aux années du centrisme", c'est-à-dire de l'alliance entre démocrates-chrétiens, sociaux-démocrates, républicains et libéraux qui avait caractérisé la première législature.

Trois éléments de nouveauté, deux relevant de la politique nationale et un d'origine strictement universitaire, ont contribué à modifier profondément le monde étudiant à partir de la seconde moitié des années 1950, en le rendant toujours plus attentif aux priorités de la vie publique "adulte», et en le liant aux partis beaucoup plus strictement que ce qui s'était passé auparavant: le problème de l'ouverture à gauche, la mutation du Parti libéral commencée sous la direction de Giovanni 
Malagodi, la dissolution des Centres universitaires démocratiques. À partir de 1956, on commença à envisager avec de plus en plus d'insistance l'entrée du Parti socialiste dans la majorité gouvernementale. L'équilibre de la première moitié des années 1950 était rompu, et les divergences idéologiques et politiques présentes dans le monde des étudiants devinrent de plus en plus profondes et de moins en moins conciliables. La ligne dure adoptée par le Parti libéral sous la direction de Malagondi contribua à la détérioration du climat interne à l'UGI. Le Parti libéral voulait en effet exercer un contre-poids à droite au sein de l'alliance centriste, et défendre les points-clés de son programme avec énergie et détermination. Le glissement progressif du système politique italien vers la gauche et le problème de l'ouverture au Parti socialiste ont davantage encore excentré la stratégie de Malagodi, la rendant importune et perturbatrice. La scission des radicaux du PLI, qui eut lieu en 1955, introduisit à l'intérieur de la «famille » libérale un conflit politique et personnel très dur.

Il était impossible que l'importante convergence goliardique, engagée comme elle était en politique et liée étroitement surtout aux libéraux et aux sociaux-démocrates, riche de militants de parti à son sommet, soit épargnée par ces tensions. D'autant plus que les nouvelles circonstances de la vie publique nationale avaient produit précisément dans le domaine de l'Université une transformation d'une importance telle qu'elle introduisit un nouveau motif de dissension et de division : la dissolution des CUDI. Depuis la fin des années 1940, la croissance politique et électorale de l'Union goliardique avait gagné sur l'espace stratégique et sur l'influence des CUDI - qui étaient une émanation étudiante de la Fédération des jeunes communistes tout en incluant aussi des socialistes - et les avait réduits à n'être plus grand chose. Peu satisfaits bien sûr de cette positions isolée et marginale, les étudiants du PCI avaient vu dans l'affaiblissement des tensions de la Guerre froide l'occasion de rentrer dans le jeu, et au début de 1955, avaient décidé que les CUDI devaient être dissous et que ses membres entreraient dans les associations goliardiques. D'emblée cependant, cette opération avait entrainé chez les goliardi une discussion très animée et l'occasion d'une profonde dissension. La décision d'accueillir ou non les étudiants communistes avait en réalité pris un sens plus profond, en devenant le symbole de l'affrontement politique alors en œuvre dans l'Union entre une aile "modérée ", plus ou moins strictement liées au PLI, mais souvent aussi au PSDI, et une aile au contraire plus progressiste, à laquelle appartenaient les jeunes de la gauche libérale qui étaient passés au Parti 
radical et où, tant du point de vue quantitatif que qualitatif, les étudiants inscrits au PSI étaient de plus en plus fortement influents.

Jusqu'en 1955, les étudiants socialistes étaient alliés aux communistes à l'intérieur des CUDI. Ils étaient ensuite tombés d'accord pour dissoudre les Centres, et avaient eux aussi décidé d'entrer dans l'UGI. Les associations goliardiques les avaient accueillis sans aucune difficulté, et en leur sein, les jeunes des CUDI avaient pu s'unir aux étudiants de convictions socialistes qui y militaient déjà, qui s'étaient éloignés du PSI parce qu'ils n'en partageaient pas la position frontiste, mais qui s'en rapprochaient désormais avec le changement de contexte historique et politique. La fraction socialiste interne à l'Union s'était renforcée davantage grâce au changement générationnel qui, dans la seconde moitié des années 1950, avait porté au sommet de l'association un nouveau groupe. Enfin, elle avait trouvé un interlocuteur de plus en plus attentif et réactif dans le Mouvement de la jeunesse du PSI qui, à partir de 1953, s'était lancé dans une stratégie de renforcement, avait timidement commencé à prendre ses distances vis-à-vis du parti «adulte », et avait décidé d'aborder sérieusement la question étudiante. Le nombre croissant de socialistes avait changé en profondeur les équilibres internes à l'univers goliardique, et surtout, combiné avec l'agressivité du PLI de Malagodi, avait contribué à en élargir le fossé idéologique interne. En bref, si dans la première moitié des années 1950, les étudiants libéraux, sociaux-démocrates et républicains pouvaient tous se retrouver sur une plate-forme centriste, dans la seconde moitié de la décennie, les jeunes radicaux et socialistes qui soutenaient sabre au clair l'ouverture à gauche ne pouvaient plus cohabiter avec les jeunes libéraux qui la rejetaient férocement. Dans ces conditions, il était inévitable que s'ouvrît une lutte pour le contrôle politique de l'Union - dont la question communiste devint le symbole et le prétexte - et qu'à cette lutte participassent également les partis «adultes», avec leurs logiques, leurs priorités et leurs ressources matérielles.

Je me limiterai à quelques considérations générales pour ce qui est de l'histoire de l'UGI et de ses rapports avec la politique entre la fin des années 1950 et la première moitié des années 1960. D’abord, durant ces années, l'histoire de l'UGI fut une histoire de scissions. La plus importante est survenue à l'été 1958 et a entraîné la naissance de l'Association nationale des goliardi indépendants (AGI), non exclusivement libérale mais à tout le moins liée à double titre au PLI en général et à Malagodi en particulier. En 1961, les Groupes d'action goliardique (GAG) quittent l'UGI, et l'AGI se scinde en une aile «droite » et une aile «gauche». Ensuite, l'Union goliardique glisse de plus en plus 
à gauche. À la fin des années 1950, en son sein, il n’y avait plus guère que les socialistes «autonomistes», c'est-à-dire convaincus que le PSI devaient se détacher du PCI, et les radicaux; avant la fin des années 1960, elle comprenait des étudiants venus des trois partis de la gauche marxiste - communistes, socialistes et sociaux-prolétariens ${ }^{3}-$, et les socialistes «lombardiens », c'est-à-dire la gauche du PSI, en constituaient le centre de gravité.

Enfin et surtout, les goliardi, et avec eux tout l'espace public étudiant, s'imprégnèrent de plus en plus des priorités de la vie politique «adulte», soit que ces priorités aient été directement transmises par les partis, soit que la vie publique étudiante les aient adoptées de façon autonome. Communistes et socialistes de gauche restaient attachés à une idée syndicale de la représentation étudiante qui leur offrait divers avantages : la possibilité de trouver des éléments de convergence avec les catholiques, et donc de ne pas de nouveau s'isoler comme à l'époque des CUDI ; le moyen, d'autre part, d'éviter que la convergence intervienne sur un projet politique réformiste, et de pousser ainsi la vie politique universitaire à proposer une rupture avec le capitalisme. Les radicaux de gauche, privés d'un solide appui partisan en dehors des campus, faisaient tout pour se maintenir au centre de la politique étudiante, en soutenant généralement la conception syndicale de la gauche marxiste - conception qu'ils avaient eux-même élaborée à l'origine - mais en tentant dans le même temps d'éviter que les socialistes et les communistes prennent le dessus, et en se montrant ainsi également disponibles pour une orientation inverse. Socialistes autonomistes, sociaux-démocrates, républicains, libéraux progressistes et une partie des radicaux, qu'ils soient membres de l'UGI ou de la gauche de l'AGI, entendaient trouver un accord avec les démocrates-chrétiens de l'Entente sur un projet politique analogue à celui du centre-gauche et qui l'emporta au niveau national entre 1962 et 1964. La fraction emmenée par Malagodi de la goliardia indépendante voulait enfin s'opposer à cette alliance.

Parce qu'ils en suivaient les logiques et participaient à leurs combats, à partir de la fin des années 1950, les goliardi, et en général les étudiants, virent se renforcer leurs liens avec les partis «adultes». Et ce, dès le début, car pour faire vivre les associations, il faut de l'argent, et les partis représentaient pour beaucoup d'entre elles la principale source de financement. Le rapport entre étudiants et forces politiques reposait ainsi

\footnotetext{
${ }^{3}$ Le Parti socialiste italien d'unité prolétarienne (PSIUP) est né en janvier 1964 de la scission de la gauche du Parti socialiste, s'opposant à la participation socialiste dans le gouvernement de centre-gauche.
} 
d'emblée sur un déséquilibre, et l'autonomie des jeunes devenait un luxe que seule la bonne volonté ou le calcul intéressé des «adultes » pouvaient concéder. Cependant, sauf dans quelques cas, je ne crois pas que l'on puisse parler d'une véritable subordination des associations étudiantes ou d'aliénation de leurs directions. Ce qui est certain, c'est que la vie publique des campus finit par s'identifier presque intégralement avec la vie nationale: les étudiants poursuivaient des finalités politiques l'autonomie socialiste, le centre-gauche, la troisième force - qui n'avaient rien à voir avec les problèmes spécifiques de l'Université. Cependant, en poursuivant ces buts, loin d'être de purs exécutants de plans conçus sans eux, les étudiants proposaient souvent leurs propres projets. Forts de ces propositions, ils discutaient ensuite avec les instances nationales, en agissant de concert avec les partis, ou, le plus souvent, avec les courants de ces partis. Les racines du manque d'autonomie de la vie publique des étudiants ne doivent donc pas tant être cherchées dans les pressions que les partis pouvaient exercer de l'extérieur, que dans l'excès de politique des dirigeants étudiants, tout à leur désir de jouer les premiers rôles à la table de la « grande » politique. C'est, partant de ces prémisses, qu'il faut ensuite comprendre, au cas par cas, et en faisant attention aux nuances pas toujours faciles à interpréter, à quel moment les choix politiques des étudiants étaient réellement la répétition de choix faits ailleurs et à quel moment c'était le fruit d'un projet autonome mais «organiquement» relié à un projet plus vaste; à quel moment enfin, ces choix provenaient exclusivement des étudiants, avant d'obtenir, seulement dans un deuxième temps, l'appui d'une organisation « adulte ».

À côté de l'histoire politique de la vie publique étudiante, il existe toujours une histoire idéologique. Et sur ce plan, les principes qui prévalaient au tout début de l'UGI continuaient à séparer ce qui était bon pour les campus de ce qui ne l'était pas. L'indépendance farouche des étudiants était à chaque fois revendiquée à chaque occasion offerte par la vie publique des universités comme étant une valeur et une donnée des événements de la vie associative et représentative : congrès, communiqués de presse, documents programmatiques. Chaque groupe revendiquait cette indépendance pour lui-même, tout en tentant de délégitimer les groupes et stratégies concurrents en les accusant d'introduire sur les campus des thématiques et des intentions qui leur étaient étrangères. Enfin, preuve définitive de la sincérité de ce qui était dit, on retrouve ce même type de discours dans les documents privés des dirigeants étudiants. De cette manière cependant, la rhétorique légitimatrice de l'espace publique étudiant se heurtait frontalement à la vie quotidienne réelle des associations et des instances représentatives. 
En théorie, les étudiants affirmaient leur autonomie, et déclaraient vouloir suivre une logique spécifique dictée par les exigences de la vie de campus; en pratique, ils faisaient de la politique en suivant scrupuleusement l'agenda idéologique fixé au niveau national. C'est dans cette contradiction que l'on doit, à mon avis, chercher au moins quelques-unes des causes de la tempête qui, en 1968, balaya les associations et les instances représentatives étudiantes : les mouvements renvoyèrent aux organisations étudiantes traditionnelles les valeurs qu'elles avaient elles-mêmes soutenues et revendiquées, et qu'elles s'étaient pourtant montrées incapables de respecter.

\section{Le système politique italien et les événements de 1968}

En Italie, 1968 ne naît pas du néant. L'émergence, parmi les étudiants, de regroupements particulièrement radicaux et hostiles aux institutions estudiantines remonte en réalité à 1962 environ. Durant quelques années, grosso modo jusqu'en 1967, ces tendances parvinrent toutefois à exister au sein des associations et des organismes représentatifs, dans un dialogue parfois tendu mais toujours contrôlé, sur l'opportunité, les moyens et les temps d'une réforme profonde des mécanismes de la vie publique étudiante. Étant donné l'étroite corrélation entre politique étudiante et politique nationale, durant ces années, l'extrémisme et l'agitation des jeunes se renforcèrent aussi à l'intérieur des partis, en particulier au PCI et au PSIUP, comme dans le monde catholique. Même dans ce contexte cependant, il n'y eut pas de problèmes particuliers de cohabitation. Dans les universités, les courants qui déboucheraient sur la contestation de 1968, ou qui s'y rallieraient, tirèrent leur force et leur légitimité d'au moins deux types de phénomènes. D'un point de vue culturel, des thèmes tels que l'autonomie, le spontanéisme, l'originalité, la créativité des jeunes, l'opposition aux institutions des «adultes », trouvaient plus d'un écho dans le répertoire de valeurs élaboré au début des années 1950 par la "première » goliardia. Les intentions et les raisonnements des jeunes les plus radicaux et les plus agités n'étaient donc en rien étrangers à la tradition des associations et des organismes représentatifs des étudiants. Ils pouvaient même s'appuyer sur elles. D'un point de vue matériel ensuite, les premières années de la décennie 1960 ont vu l'émergence de revendications étudiantes de base, "techniques » et syndicales, que les instances représentatives peinaient à contrôler et à exprimer. Il convient de remarquer que l'extraordinaire changement démographique et social qui modifia radicalement le profil des institutions universitaires italiennes 
n'a pas seulement été difficilement géré par la classe politique «adulte », mais a mis en difficulté jusqu'aux dirigeants étudiants qui, du reste, partageaient les priorités de la première. Les occupations des facultés d'architecture en 1963 représentèrent, de ce point de vue, le premier signal d'alarme. Les étudiants d'architecture étaient mus par une aspiration «corporative » : le désir de réformer l'organisation spécifique de leur faculté et de leurs études. L'aspect technique prit cependant très vite une signification politique plus générale, tandis que le choix d'occuper les facultés signifiait bien sûr le refus de faire passer leurs revendications à travers les organismes représentatifs et témoignait ainsi de la crise de ces derniers.

Face à ce double processus de radicalisation, autant culturel que syndical, les associations et les organismes représentatifs traditionnels réagirent en déplaçant de plus en plus leur centre de gravité idéologique vers des positions d'agitation politique. Le «spontanéisme » étudiant fut porté - d'un côté - jusqu'à ses conséquences institutionnelles les plus extrêmes: la critique des mécanismes représentatifs de l'UNURI par exemple, et le désir d'élargir et de renforcer la vie politique étudiante à travers des formes d'assemblées de démocratie directe. D’un autre côté, conséquence évidente de la crise du centrisme d'abord et de la faillite du centre-gauche ensuite, le «spontanéisme» fut plus explicitement que par le passé lié à une perspective d'alternative au «système» d'origine marxiste au sens large: les étudiants assimilés aux travailleurs; la conviction que les étudiants devaient se rattacher organiquement et en permanence à la classe ouvrière; la volonté que ce nouveau bloc social s'oppose de manière plus ou moins intransigeante à l'ordre économique et politique du pays. Ces éléments d'innovation culturelle devaient ensuite déboucher sur une profonde réforme des associations et des organismes représentatifs : une réforme qui - envisagée justement pour satisfaire les demandes de la «base» syndicale et des courants les plus radicaux - transformerait l'orientation avant tout politique des instances étudiantes en une orientation avant tout syndicale; une réforme qui valoriserait les facultés par rapport aux universités et qui introduirait des formes de démocratie directe et affaiblirait les mécanismes de délégation.

Toutefois, dans les faits, le caractère politicien et l'adaptation aux logiques de l'espace public « adulte » que les associations et représentations étudiantes avaient acquis au tournant de la décennie ne changèrent pas du tout au cours des années 1960. Le poids de la situation politique complexe qui caractérisa la première législature du centre-gauche «organique » (1963-68) devint même de plus en plus sensible dans les campus, et par conséquent, les pressions que les fédérations des jeunes des partis 
des partis exercèrent sur les étudiants se firent beaucoup plus brutales et directes. Dans ces circonstances, même les larges débats - structurés, récurrents et répétitifs - sur la nécessité de réformer les associations et l'UNURI, et sur la façon de les réformer, finirent par être subordonnés à la lutte entre partis et courants des partis, et se conclurent par un échec. À la demande d'autonomie et de renouvellement qui venait d'« en bas », les structures traditionnelles de la vie publique étudiante donnèrent ainsi une réponse purement rhétorique, aggravant de ce fait leur crise: en l'absence d'une pratique politique qui l'accompagnât, leur course idéologique vers l'agitation élargit davantage le fossé entre la théorie de la politique étudiante et sa réalité ; et en l'absence de réformes effectives, la discussion prolongée sur les réformes légitima ceux qui pensaient que les associations et les organismes représentatifs étaient désormais totalement inadaptés et devaient être balayés.

Les tensions radicales qui étaient apparues au début des années 1960 et qui déboucheraient ensuite sur la vague de contestation de 1968 puisèrent énergie et consistance dans ce hiatus croissant entre idéologie et réalité. Dans de nombreux cas, ce hiatus fut même leur berceau. Un regard même superficiel sur les dernières années des associations et des organismes représentatifs démontre en effet clairement comment se sont créées en leur sein de fortes minorités que les directions respectives ne parvenaient déjà plus ni à éliminer, ni à contrôler, ni à absorber. Par exemple, ce n'est pas un hasard si l'un des plus importants documents de la "préhistoire » de 1968, le Projet de thèse du syndicat étudiant élaboré en février 1967 par les occupants de l'université de Pise, est né dans le milieu goliardique et a recyclé, en les radicalisant, les idées et les projets qui circulaient déjà depuis quelques années à l'UGI. Comme il est significatif qu'au sommet de l'Union composé de socialistes et communistes, on ait éprouvé les pires difficultés à battre la minorité agitatrice, quelques mois plus tard, en mai 1967, lors du XVI ${ }^{\mathrm{e}}$ congrès de l'UGI.

Le raisonnement sur le fossé entre ambitions théoriques et réalisations pratiques, entre radicalisme idéologique et pragmatisme effectif peut être appliqué également au système politique italien d'alors. À partir de la fin des années 1950, l'aspiration non réalisée mais persistante à une profonde transformation politique et sociale du pays que la République italienne avait héritée de la Résistance, trouva un nouvel élan, d'abord avec la crise politique de l'été 1960 et la relance d'un antifascisme resté marginal pendant toute la décennie précédente, puis surtout - avec l'entrée des socialistes dans la majorité gouvernementale. Cette profonde transformation culturelle, qui consista formellement en l'affirmation d'une forma mentis progressiste de nature plus ou moins 
radicale, ne trouva cependant qu'un semblant de réalisation dans un ordre politique et une action gouvernementale bien trop prudents. Le centre-gauche coexista ainsi avec des attentes de régénération nationale auxquelles il se montra incapable de répondre. L'effort de réforme qu'il accomplit pourtant se révéla inadapté, non seulement et pas tant en soi ou en regard des exigences d'un pays en cours de modernisation accélérée et désordonnée, mais en regard des espoirs qu'il avait engendrés et dont il était le fruit, espoirs qui avaient fait de la palingénésie rhétorique de l'antifascisme l'idéologie officielle du système des partis.

Dans les années 1960, les aspirations à la rénovation complète du pays, implicites dans les racines résistancialistes de la République et plus affirmées dans la conscience que la politique étudiante avait d'elle-même, se présentèrent en outre sous des formes idéologiques renouvelées. D’un côté, ce fut simplement un processus de radicalisation supplémentaire. Mais il ne s'agit pas seulement de cela. Le programme originel que, dans la première moitié des années 1950, les goliardi avaient réussi à imposer aux organismes représentatifs et aux autres associations était un programme de « révolutionnarisme libéral », fondé sur le désir d'institutions plus ouvertes et plus démocratiques et d'une vie culturelle plus dynamique et innovatrice. C'était en somme un programme progressiste inscrit dans les limites d'une conception «classique » de la modernité. À partir des années 1960, ce sont au contraire les idées mêmes d'institution, de culture et de modernité qui étaient radicalement remises en cause. Le « révolutionnarisme libéral » se transforma ainsi en un révolutionnarisme tout court, qui n'avait plus rien de libéral, et à la rigueur était surtout socialiste, mais d'un socialisme décliné sur une forme activiste, syndicaliste, révolutionnaire en somme, et non sous la forme du matérialisme historique, et encore moins du positivisme.

L'interprétation de 1968 que je propose, selon laquelle l'insurrection étudiante aurait été engendrée aussi par la rébellion de l'idéologie de la République contre sa pratique politique, introduit un élément de nouveauté par rapport aux recherches historiographiques et politistes qui ont cependant mis en évidence des éléments de continuité entre la contestation étudiante et le contexte dans lequel elle s'est développée. Ces études se sont principalement focalisées sur deux causes ou prémices de 1968 : l'émergence dans les années 1960 d'un nouveau radicalisme essentiellement (mais pas seulement) jeune, visible dans les partis, dans le débat intellectuel et dans la rue; l'incapacité du système politique, par conservatisme, par rigidité, par excès de politique politicienne, d'affronter et de désamorcer ce défi. Sans vouloir nier la présence ni l'importance 
de ces composantes, je pense qu'il faut ajouter un autre élément: les origines de la contestation étudiante doivent être recherchées non seulement aux marges ou dans l'inadaptation du système politique, mais aussi en son cœur idéologique, précisément dans l'esprit radical que ce système acquit dès sa naissance, et dans la résurgence de cet esprit au lendemain de la «parenthèse » centriste, et en concomitance non fortuite, avec la genèse du centre-gauche. Un esprit radical, en outre, qui dans l'histoire des associations et des instances représentatives étudiantes apparaît particulièrement fort, et donc particulièrement «trahi » à la fin des années 1950 et 1960, et particulièrement revendiqué par 1968.

Entre contestation étudiante et système politique, il y eut donc sous certains aspects, non pas excès de divergence mais de convergence. Convergence sur le plan des valeurs et de l'idéologie, si bien que devant la montée de la protestation, les institutions furent non pas trop rigides, mais trop souples, et dans les faits, ne s'opposèrent pas à leur propre déligitimation, la facilitèrent même. Pas seulement par esprit tactique fondé sur la nécessité de garantir avant tout l'ordre public. Mais aussi par conviction, présente dans une grande partie de la classe politique officielle, que de nombreux traits culturels proposés par l'insurrection des étudiants appartenaient au patrimoine génétique de la République, et devaient pour cette raison être récupérés, sans doute en doses homéopathiques plutôt qu'à travers le remède radical proposé par les étudiants. Pour cela également, parce que la protestation étudiante s'enracinait en partie dans le ventre de la tradition politique républicaine, en Italie, 1968 ne rencontra pas une réaction d'opposition politique et culturelle dure, «à la française ». La contestation fut habile à retrouver ces consonances profondes et les utilisa jusqu'à revendiquer pour soi une cohérence systémique. Cette habileté, combinée à l'absence d'une réaction forte de la part du système politique officiel, doit ainsi être considérée comme l'une des principales causes du fait que, à la différence de ce qui arriva ailleurs, 1968 se prolongea en Italie pendant presque toute la décennie 1970. L' «étirement» de 1968 jusqu'à 1977 peut en somme être considéré à la fois comme le produit de la rencontre entre la stratégie inclusive provenant du système, qui cherchait à récupérer les valeurs du mouvement en les dissociant de leur radicalisme formel, et comme le projet de la partie la plus intransigeante du mouvement, qui essayait de sauver la perspective de la révolution en la séparant de l'insurrection violente et immédiate.

Tout en étant sous de nombreux aspects « fille» de la République, et tout en s'étant prolongé pendant une décennie, d'un point de vue 
institutionnel, c'est-à-dire en termes de renouvellement de la représentation politique, 1968 resta en Italie en grande partie stérile. Ce qui arriva sur les campus le démontre selon moi plutôt clairement. La contestation étudiante secoua l'UGI, l'AGI, l'Entente, l'UNURI qui, malgré leurs défauts, avaient pendant vingt ans permis aux étudiants d'être représentés dans une structure solide, structurée et garantie; elles avaient été une préparation remarquable à la vie démocratique et un non moins exceptionnel vivier de la classe politique ; elles avaient fait le lien entre l'espace public des campus et l'espace public national, en accroissant la légitimité de ce dernier. À tout cela, et bien que les critiques adressées aux institutions fussent fondées, les contestataires de 1968 ne substituèrent pas autre chose qu'une longue succession de flambées activistes, en laissant finalement le monde des étudiants totalement démembré.

\section{Bibliographie}

Aldo Agosti, Luisa Passerini, Nicola Tranfaglia (a cura di), La cultura e $i$ luoghi del '68, Milano, Franco Angeli, 1991.

Marco Boato, Il '68 è morto: viva il '68!, Verona, Bertani, 1979.

Franco Catalano, I movimenti studenteschi e la scuola in Italia (1938-1968), Milano, il Saggiatore, 1969.

Donatella Della Porta, Movimenti collettivi e sistema politico in Italia, 19601995, Roma-Bari, Laterza, 1996.

Marcello Flores, Alberto De Bernardi, Il Sessantotto, Bologna, il Mulino, 1998.

Paolo Ghione, Marco Grispigni (a cura di), Giovani prima della rivolta, Roma, Manifestolibri, 1998.

Giovanni Orsina, Il "luogo politico" del Partito liberale nell'Italia repubblicana, in Giovanni Orsina (a cura di), Il Partito liberale nell'Italia repubblicana. Guida alle fonti archivistiche per la storia del Pli, Atti dei Congressi e Consigli Nazionali, Statuti del Pli, 1922-1992, libro con Dvd-Rom, Soveria Mannelli, Rubbettino, 2004, pp. 11-59.

Giovanni Orsina, "Quando l'Antifascismo sconfisse l'antifascismo. Interpretazioni della resistenza nell'alta cultura antifascista italiana (195565) », Ventunesimo secolo, IV , 7, aprile 2005, pp. 9-43.

Giovanni Orsina, Il sistema politico italiano. Lineamenti di un'interpretazione revisionistica, in Pier Luigi Ballini, Sandro Guerrieri, Antonio Varsori (a cura di), Le Istituzioni repubblicane dal centrismo al centrosinistra (1953-1968), Roma, Carocci, 2006, pp. 309-33. 
Giovanni Orsina, Gaetano Quagliariello (a cura di), La formazione della classe politica in Europa, 1945-1956, Manduria-Bari-Roma, Lacaita, 2000.

Giovanni Orsina, Gaetano Quagliariello (a cura di), La crisi del sistema politico italiano e il Sessantotto. Una ricerca di storia orale, Soveria Mannelli, Rubbettino, 2005.

Luisa Passerini, Autoritratto di gruppo, Firenze, Giunti, 1988.

Gaetano Quagliariello, Studenti e politica. Dalla crisi della goliardia prefascista al I congresso nazionale universitario (1925-1946), Manduria-Bari-Roma, Lacaita, 1987.

Gaetano Quagliariello, "Parcours de formation de la classe politique italienne : les étudiants dans la transition du fascisme à la démocratie », Revue d'bistoire moderne et contemporaine, vol. 45, n¹, 1998, pp. 196-226.

Gaetano Quagliariello (a cura di), La politica dei giovani in Italia (19451968), Roma, Luiss University Press, 2005.

Sidney G. Tarrow, Democrazia e disordine. Movimenti di protesta e politica in Italia, 1965-1975, Roma-Bari, Laterza, 1990.

Giuliano Urbani, Politica e universitari, Firenze, Sansoni, 1966. 